\title{
Photo-mediated Seedless Synthesis of Silver Nanoparticles Using CW-Laser and Sunlight Irradiation
}

\author{
F. Félix-Domínguez ${ }^{1}$, R. C. Carrillo-Torres², J. A. González ${ }^{1}$, J. Hernández-Paredes ${ }^{2}$, R. Sánchez- \\ Zeferino $^{2}$, M. E. Álvarez-Ramos ${ }^{2}$. \\ 1. Doctorado en Nanotecnología, Departamento de Física, Universidad de Sonora, Hermosillo, Sonora, \\ México. \\ 2. Departamento de Física, Universidad de Sonora, Hermosillo, Sonora, México.
}

Noble metal nanoparticles, such as silver nanoparticles (AgNPs), have found technological applications due to their localized surface plasmon resonance (LSPR). In this context, a great quantity of synthetic methods for the preparation of AgNPs have been developed, including photo-chemical synthesis [1]. The photo-chemical synthesis is an advantageous technique because it is simple and environmentally friendly [2]. In this work, synthesis of AgNPs was performed via direct photo-reduction process of the silver nitrate and sodium citrate solutions without the previous addition of silver seeds.

Silver nanoparticles were synthesized in aqueous solution using a mixture of silver nitrate $(5 \mathrm{mM})$ as metallic precursor and sodium citrate $(3 \mathrm{mM})$ as reducing agent. $300 \mu \mathrm{L}$ of reaction mixture was placed in a polystyrene cuvette and was irradiated with laser light $(\lambda=488 \mathrm{~nm}, 130 \mathrm{~mW})$ from an Ar ion CWLaser (Melles-Griot, USA) or direct sunlight (near noon) varying irradiation time from 5 to 60 minutes. Both synthesis took place at $\sim 20^{\circ} \mathrm{C}$. Scanning Transmission Electron Microscopy (STEM) analysis was carried out with a field emission microscope JEOL JSM-7800F, operated at $30 \mathrm{kV}$.

The synthesis is achieved by photo-oxidation of sodium citrate to acetone-1,3-dicarboxylate, reducing ionic silver to $\mathrm{Ag}^{0}$ in the process. Furthermore, acetone-1,3-dicarboxylate can be chemisorbed on the nanoparticle surface controlling their growth and also stabilizing the nanostructures in the solution [3]. Therefore, the only residual product is $\mathrm{CO}_{2}$, which makes of this synthetic route a clean technique.

In all cases laser synthesized AgNPs showed high size polydispersity. The size of the NPs ranges from a few nanometers $(\sim 10 \mathrm{~nm})$ up to more than $100 \mathrm{~nm}$, forming large aggregates with irregular morphology (Figure 1). Moreover, it can be seen that as the irradiation time elapses there is a decrease in the number of the smaller NPs. It is worth to mention that the agglomeration of NPs hampered to obtain the size distribution curve.

Sunlight mediated AgNPs showed better size distribution and morphologies than that obtained by laser irradiation. The size of the nanoparticles increased with the increase of the irradiation time (Figure 2 a-c). It is proposed that the improvement in size and morphology is achieved thanks to the uniform illumination over the reaction cuvette when using sunlight. It is important to note that the laser beam passes through a small volume of reaction mixture causing a localized reaction in the sample. Sunlight mediated synthesis overcomes the later, so we can assume that all the solution receives the same quantity of light in every point at the same time, inducing a uniform nucleation and growth of the nanoparticles in the solution. In order to study the effect of silver/citrate ratio on the size and morphology of the NPs, we performed an experiment using a lower silver nitrate concentration $(0.1 \mathrm{mM})$ keeping constant the sodium citrate concentration. This solution was exposed to 60 minutes of sunlight. Figure $2 \mathrm{~d}$ shows the obtained nanoparticles. In this case, it is possible to observe small uniform spheroidal nanoparticles and large plate- 
like nanoparticles, which were not observed in the experiments with higher silver concentration (figure 2c).

In conclusion, the sunlight synthesis allowed us to have better control of the size and morphology of AgNPs than the laser irradiation synthesis. Irradiation time was also an important parameter to consider for controlling these features. The silver:citrate ratio played an important role defining the final morphology of the nanostructures [4].

\section{References:}

[1] B Wiley et al., Chemistry A European Journal 11 (2005), p. 454.

[2] M Sakamoto, M Fujistuka, T Majima, Journal of Photochemistry and Photobiology C:

Photochemistry Reviews 10 (2009), p. 33.

[3] A Henglein, M Giersig, The Journal of Physical Chemistry B 103 (1999), p. 9533.

[4] The authors acknowledge funding from Consejo Nacional de Ciencia y Tecnología (CONACyT), project number 255791-INFR-2015.
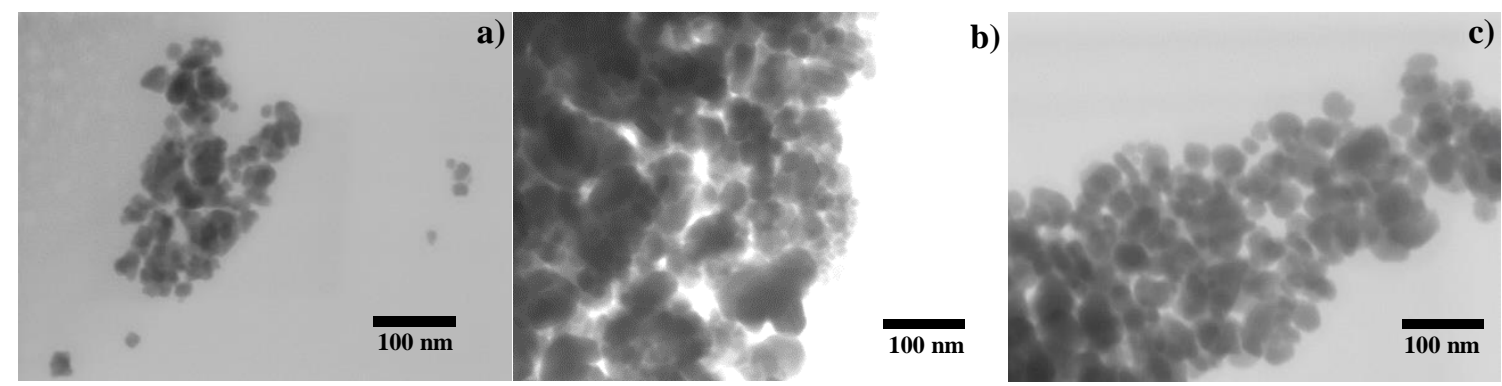

Figure 1. AgNPs synthesized using CW laser irradiation (488 nm, $130 \mathrm{~mW}$ ) varying irradiation time a) 10 minutes, b) 15 minutes and c) 60 minutes.

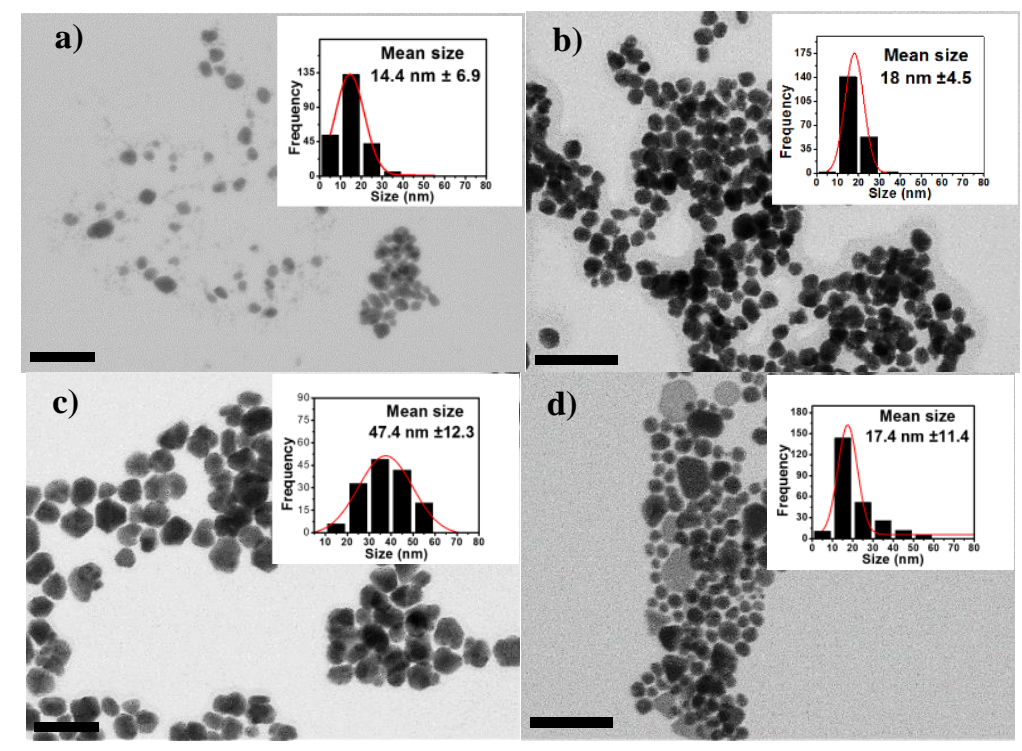

Figure 2. AgNPs synthesized by sunlight irradiation varying irradiation time: a) 5 minutes, b) 15 minutes and c) 60 minutes. d) AgNPs prepared with $0.1 \mathrm{mM} \mathrm{AgNO}_{3}$ and the same sodium citrate concentration, irradiated with sunlight during 60 minutes. Insets show size distribution histograms. Scale bars represent $100 \mathrm{~nm}$. 